\title{
A REPRESENTATION FORMULA FOR HARMONIC FUNCTIONS
}

\section{CHIN-HUNG CHING AND CHARLES K. CHUI}

Abstract. We give a formula to reconstruct certain entire harmonic functions from their values on some lattice points.

1. Introduction and results. In [1], Boas proved the following uniqueness theorem for harmonic functions.

THEOREM A. Let $u(z)$ be a real-valued entire harmonic function of exponential type less than $\pi$ such that $u(m)=0$ and $u(m+i)=0$ for $m=0$, $\pm 1, \pm 2, \cdots$. Then $u(z) \equiv 0$.

$\mathrm{He}$ also asked if it is possible to reconstruct an entire harmonic function of exponential type less than $\pi$ from its values on the lattice points $m$, $m+i$. We have

THEOREM 1. Let $u(z)$ be a real-valued entire harmonic function of exponential type $\tau \leqq \pi$ such that $u(x)$ and $u(x+i)$ are in $L^{2}(-\infty, \infty)$. Then

$$
\begin{aligned}
u(z)= & u(x+i y) \\
= & \sum_{n=-\infty}^{\infty} \frac{u(n)}{2 \pi} \int_{-\pi}^{\pi} \frac{\sinh t(1-y)}{\sinh t} e^{i t(x-n)} d t \\
& +\sum_{n=-\infty}^{\infty} \frac{u(n+i)}{2 \pi} \int_{-\pi}^{\pi} \frac{\sinh t y}{\sinh t} e^{i t(x-n)} d t \\
& +c_{1} e^{\pi x} \sin \pi y+c_{2} e^{-\pi x} \sin \pi y
\end{aligned}
$$

where the series converges uniformly in every strip $|y| \leqq K<\infty$. Furthermore, if $\tau<\pi$ then $c_{1}=c_{2}=0$, and if $\tau=\pi$ then

$$
c_{1}=\lim _{x \rightarrow \infty} \frac{e^{-\pi x} u(x+i y)}{\sin \pi y} \text { and } c_{2}=\lim _{x \rightarrow \infty} \frac{e^{-\pi x} u(-x+i y)}{\sin \pi y}
$$

for any $y, 0<y<1$.

Received by the editors September 20, 1972 and, in revised form, October 27, 1972. AMS (MOS) subject classifications (1970). Primary 31A10; Secondary 30A62.

Key words and phrases. Harmonic functions, entire functions of exponential type, Paley-Wiener theorem, Plancherel theorem, representation formula.

(c) American Mathematical Society 1973 
Hence, we have the following

COROLlary. Let $u(z)$ be a real-valued entire harmonic function of exponential type equal to $\pi$ such that $u(x)$ and $u(x+i)$ are in $L^{2}(-\infty, \infty)$, $u(m)=0$ and $u(m+i)=0$ for $m=0, \pm 1, \pm 2, \cdots$. Then

$$
u(z)=c_{1} e^{\pi x} \sin \pi y+c_{2} e^{-\pi x} \sin \pi y
$$

for some real constants $c_{1}$ and $c_{2}$.

Of course, the above results hold for complex-valued $u(z)$.

2. Proof of Theorem 1. Let $f(z)$ be an entire function with $\operatorname{Re} f=u$ and let $F(z)=f(z)+[f(\bar{z})]-$. Then $F(z)$ is an entire function of exponential type at most $\pi$. By the Paley-Wiener theorem, we have

$$
F(z)=\int_{-\pi}^{\pi} e^{i z t} \phi(t) d t .
$$

Since $F(x)=2 u(x)$ is in $L^{2}(-\infty, \infty), \phi(t)$ is in $L^{2}(-\pi, \pi)$ (cf. [2]). Hence,

$$
\sum_{n=-\infty}^{\infty} u^{2}(n)=\frac{1}{4} \sum_{n=-\infty}^{\infty} F^{2}(n)=\frac{1}{4} \int_{-\pi}^{\pi}|\phi(t)|^{2} d t<\infty .
$$

Similarly, we have $\sum u^{2}(n+i)<\infty$. Using Schwarz's inequality and the Plancherel theorem, we obtain

$$
\begin{aligned}
&\left\{\sum_{n=-\infty}^{\infty}\left|\frac{u(n)}{2 \pi} \int_{-\pi}^{\pi} \frac{\sinh t(1-y)}{\sinh t} e^{i t(x-n)} d t\right|\right\}^{2} \\
& \leqq\left\{\frac{1}{4} \pi^{2} \sum_{n=-\infty}^{\infty} u^{2}(n)\right\}\left\{\sum_{n=-\infty}^{\infty}\left|\int_{-\pi}^{\pi}\left\{\frac{\sinh t(1-y)}{\sinh t} e^{i t x}\right\} e^{-i t n} d t\right|^{2}\right\} \\
& \leqq c_{1} \int_{-\pi}^{\pi}\left|\frac{\sinh t(1-y)}{\sinh t} e^{i t x}\right|^{2} d t \\
& \leqq c_{2} \int_{-\pi}^{\pi}\left|\frac{\sinh t(1-y)}{t}\right|^{2} d t \leqq c_{3}|1-y|^{2} e^{2 \pi|y|}
\end{aligned}
$$

for all $x+i y$. Similarly, we have

$$
\sum_{n=-\infty}^{\infty}\left|\frac{u(n+i)}{2 \pi} \int_{-\pi}^{\pi} \frac{\sinh t y}{\sinh t} e^{i t(x-n)} d t\right| \leqq c_{4}|y| e^{\pi|y|} .
$$

Hence, the series in (1) converges uniformly in every strip $|y| \leqq K<\infty$ to a real-valued entire harmonic function $w(z)$ with

$$
|w(x+i y)| \leqq(\alpha+\beta|y|) e^{x|y|}
$$

for some constants $\alpha, \beta$ and for all $x+i y$. 
Since $F(z)$ is an entire function of exponential type $\leqq \pi$ and its restriction to the real axis is in $L^{2}(-\infty, \infty)$, we have

$$
\begin{aligned}
u(x) & =\frac{1}{2} F(x)=\frac{1}{2 \pi} \sum_{n=-\infty}^{\infty} \frac{\sin \pi(x-n)}{x-n} F(n) \\
& =\frac{1}{\pi} \sum_{n=-\infty}^{\infty} \frac{\sin \pi(x-n)}{x-n} u(n) \\
& =\sum_{n=-\infty}^{\infty} \frac{u(n)}{2 \pi} \int_{-\pi}^{\pi} e^{i t(x-n)} d t=w(x)
\end{aligned}
$$

for all real $x$. Similarly, we can also conclude that $u(x+i)=w(x+i)$.

Now, let $h(z)$ be an entire function with $\operatorname{Re} h=u-w$. From (2) and Carathéodory's inequality, we have

$$
h(z)=O\left(e^{(\pi+\varepsilon)|z|}\right)
$$

for $0<\varepsilon<1$. Let $H(z)=h(z)+[h(\bar{z})]-$. Then $H(x)=2 \operatorname{Re} h(x)=0$ for all real $x$ so that $H(z) \equiv 0$ or

$$
h(z)=-[h(\bar{z})]-.
$$

Similarly, let $G(z)=h(z+i)+[h(\bar{z}+i)]-$. Then $G(x)=2 \operatorname{Re} h(x+i)=0$ for all real $x$ so that $h(z+i)=-[h(\bar{z}+i)]-$ for all $z$. Hence, by combining this with (4), we see that $h(z)$ has period $2 i$. Now, it is well known that an entire function with period $2 i$ satisfying (3) must be an exponential sum of the form $h(z)=\alpha+\beta e^{\pi z}+\gamma e^{-\pi z}$. That is, we have

$$
\begin{aligned}
& u(x+i y)-w(x+i y) \\
& =a+b_{1} e^{\pi x} \cos \pi y+c_{1} e^{\pi x} \sin \pi y+b_{2} e^{-\pi x} \cos \pi y+c_{2} e^{-\pi x} \sin \pi y
\end{aligned}
$$

for some real constants $a, b_{1}, b_{2}, c_{1}, c_{2}$. Since $u(x)=w(x)$ for all real $x$, $a=b_{1}=b_{2}=0$. From (2), we can conclude that

$$
c_{1}=\lim _{x \rightarrow \infty} e^{-\pi x} \frac{u(x+i y)-w(x+i y)}{\sin \pi y}=\lim _{x \rightarrow \infty} e^{-\pi x} \frac{u(x+i y)}{\sin \pi y}
$$

for $0<y<1$. Similarly, for $0<y<1$,

$$
c_{2}=\lim _{x \rightarrow-\infty} e^{\pi x} \frac{u(x+i y)}{\sin \pi y} .
$$

It is obvious that $c_{1}=c_{2}=0$ if $\tau<\pi$. 


\section{REFERENCES}

1. R. P. Boas, Jr., A uniqueness theorem for harmonic functions, J. Approximation Theory 5 (1972), 425-427.

2. - Entire functions, Academic Press, New York, 1954. MR 16, 914.

Department of Mathematics, University of Melbourne, Parkville, Australia

Department of Mathematics, Texas A\&M University, College Station, TEXAS 77843 